\title{
Research on Nuclear Sewage Diffusion of Fukushima in Japan
}

\author{
Xu Yong \\ ${ }^{1}$ Technical department in 61936 Unit of PLA, Haikou, China \\ axuyonghk@126.com, ${ }^{\mathrm{b}} \mathrm{GJhk@yahoo.com.cn}$
}

Keywords: Nuclear sewage; Ocean circulation; Impact analysis; Fukushima in Japan; Transport and diffusion.

\begin{abstract}
March 11, 2011, the Richter 9.0 earthquake (defined by Japan Meteorological Agency) occurred in the east of Japan, the epicentre was located at $38.1^{\circ} \mathrm{N}, 142.6^{\circ} \mathrm{E}$. tsunami triggered by the Earthquake is claimed up to 23 meters. The combination effect of earthquake and tsunami lead to the Fukushima nuclear power plant accident. Some water with nuclear sewage is dumped into the sea near Fukushima during the accident. This paper studies the propagation path of nuclear pollutants through ocean circulation. Climate state circulation data, assimilation re-analysis products, surface drifting buoys trajectories, three-dimensional numerical model prediction products are used in this research. The results show that nuclear pollutants have little effect on China marine environment in short time, but may cause influence in long-term.
\end{abstract}

\section{Introduction}

The sea water near Fukushima is seriously polluted by nuclear materials during the Fukushima grade 7 nuclear accidents. The 4000 times higher nuclear contamination than standard has been detected near the Fukushima coast at 30 March. The 750 times higher radioactive iodine than standard has been detected near the Fukushima coast at 2 April. From 4 April, about 10,000 tons of water polluted by nuclear materials has been discharged into the sea near Fukushima, this action lead to further pollution. The pollution has been detected 200m under the sea near the Fukushima at 30 April [1]. The nuclear pollutions can spread through the ocean circulation. We should concern if the marine environment near our country will be incurred by the disaster. This paper will focus on the analysis of nuclear pollutants spreading through ocean circulation.

\section{Data}

In this paper, the research data include climate state ocean circulation data, assimilation re-analysis products; surface drifting buoys trajectories, three-dimensional numerical model forecasting products.

(1) Climate state ocean circulation data used here are HYCOM interannual mean data. We use this data as a background to show the main ocean circulation system in the north Pacific [2].

(2) Assimilation re-analysis products used here are made by the National Marine Information Center in China. Considering the limitation of current observations, the assimilation re-analysis products may describe the three dimensional water flow information better[3].

(3) Surface drifting buoys trajectories used here are the SVP (Surface Velocity Program) surface drifting buoy observations of historical data (6378 in all) from 1979 to 2010 [4].

(4) A three dimensional regional numerical forecasting products from an ocean-atmosphere coupling model based on the WRF and POM is used during the research. The valid forecast is 7 days [5]. 


\section{Analysis and Discussion}

The Climate Ocean Current in north Pacific. The paper mainly focus on the Oyashio current and the North Pacific subtropical circulation system (Figure1), which usually includes the Kuroshio, the North Equatorial Current and the Philippines streams, etc. The upper ocean circulations are mainly forced by wind stress above the inner ocean [4]. The Oyashio flows from northeast to southwest, meanwhile Kuroshio flows from southwest to northeast. These two currents meet at the offshore of Fukushima, and then turn to east [5]. This made the Fukushima coastal and offshore water mainly flows eastward. China Sea is located in the west and upstream of the Kuroshio. The nuclear pollutions carried by ocean circulation are unlikely spread into China Sea directly within short time.

Kuroshio and its extension flowing into the North Pacific warm body are parts of the north Pacific subtropical circulation gyre (The north Pacific sub-polar circulation is not concerned here for its no direct connection with the China Sea). For the subtropical circulation, one circle will take two years at least, which means that most nuclear radioactive materials carried by the water would settle and be diluted. But some artificial radioactive materials with long half-life periods (see Tab. 1) may still reach China Sea through ocean circulation in the long-term view [6].

Table 1 Mainly radioactive materials and half-life period Table Type Styles

\begin{tabular}{ll}
\hline Name of Radioactive Material & Half-Life Period \\
\hline Lanthanum -140 & 2 days \\
\hline Iodine -131 & 8 days \\
\hline Cesium -134 & 20 years \\
\hline Cesium -137 & 30 years \\
\hline Plutonium -238 & 88 years \\
\hline Plutonium -239 & 24 thousand years \\
\hline
\end{tabular}

The numerical forecasting results. The three-dimensional numerical prediction model products lead to a similar conclusion with the former study (Fig.1). What's more, it can describe the fine structure changing every day. From this figure we can see the several clockwise and anti-clockwise meso-scale eddies near the east Japan Island. These meso-scale eddies enhance the mixing in local area, which accelerates the dilution and sedimentation process of the nuclear pollution [2].

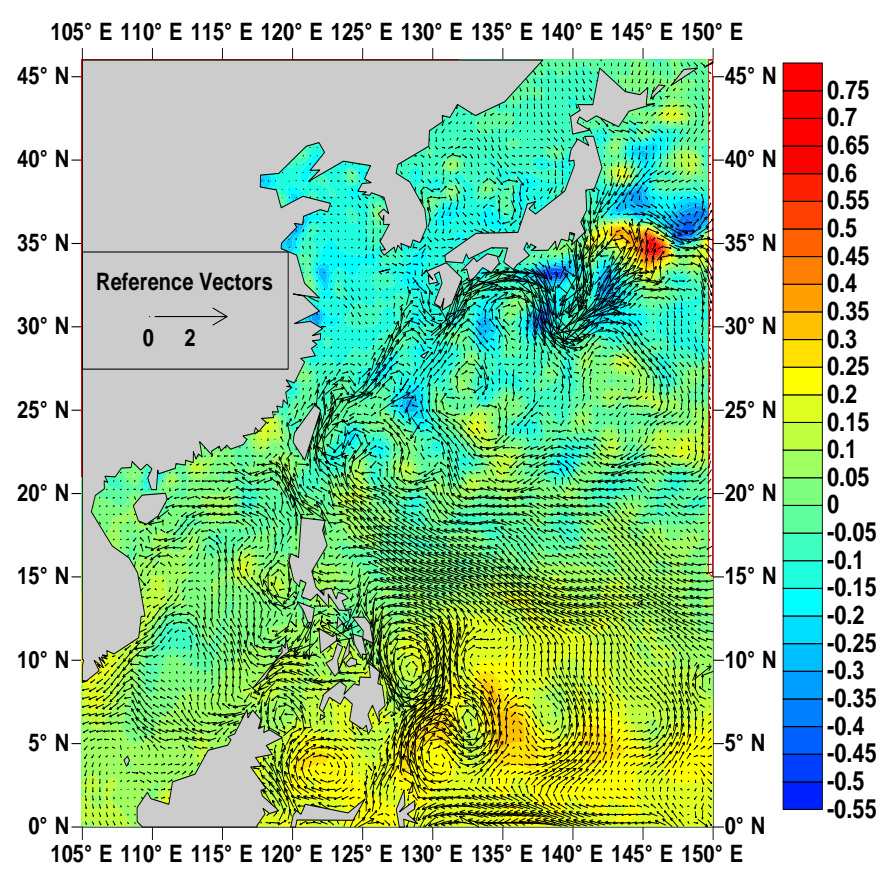

Figure. 1 Surface current (vector arrows) and satellite sea surface height anomaly (color fill chart) based on the northwest Pacific three-dimensional numerical prediction system 
To further analyze the tracks of nuclear pollution in the ocean which comes from Fukushinma, 1000 fictitious buoys were settled within $200 \mathrm{~km}$ around Fukushima coast randomly. Then we set 1000 simulated pollution tracers based on the day to day forecast surface current results from March 11 to April 5, 2011. Figure 3 shows the average forecast surface currents during March 11 - April 5, 2011 and the simulated nuclear pollution tracers. The figure shows that most of buoys move periodically near the Fukushima offshore. This movement is probably forced by tide. But a small part of buoys flow southwest along the Japan Island $[6,7]$. This part of the pollutants may be catched by the Kuroshio south of Japan and then drift eastward, or they may be southward because of the anti-cyclo vortex lead by the big bend off the Kuroshio.

The assimilation re-analysis products. In this part, we discuss the short and middle term spread of the nuclear pollutions through the assimilation re-analysis products from 1986 to 2009. One thousand assumed buoys standing for nuclear pollution are settled within 200km Fukushima coast [7], and the trajectory of buoys is tracked with the surface current(Fig. 2). Figure $2 \mathrm{a}$ is the spreading path from March 11 to April 11, 2011; Figure 2b is the spreading path from March 11 to June 11, 2011 and Figure $2 \mathrm{c}$ is the spreading path from March 11 to December 31, 2011, respect.

From the numerical tracer trajectories, we can find that the mainly directions of water movement with nuclear contamination is northeastward by 1 month, 3 months and 9 months. Thus, the scope of nuclear contamination is mainly on the east of Japan, which means that the nuclear contamination may not impact China Sea marine environmental through ocean circulation, in short period.

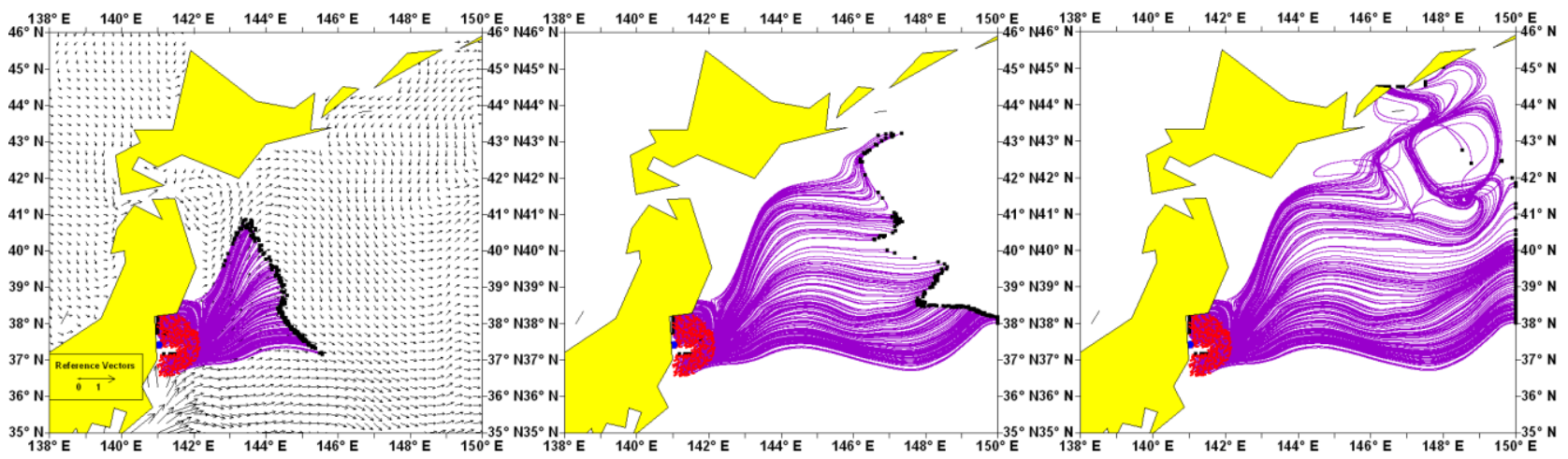

Figure. 2 Simulated the nuclear contamination tracer drift trajectory

SVP surface drifting buoy observations. In this section, we use the SVP surface drifting buoy observation data to analyze the real direction of flows around the Fukushima offshore in long-term[8]. We made a rectangular area around Fukushima, 3 degree in latitude and 4 degree in longitude (the green rectangle in Fig. 3). 176 buoys of 6378 SVP drifting buoys from 1979 to 2010 can be found in the rectangle area. The buoys tracers across the selected rectangle area are shown in figure 5 and we will discuss the spread directions of these buoys below. 


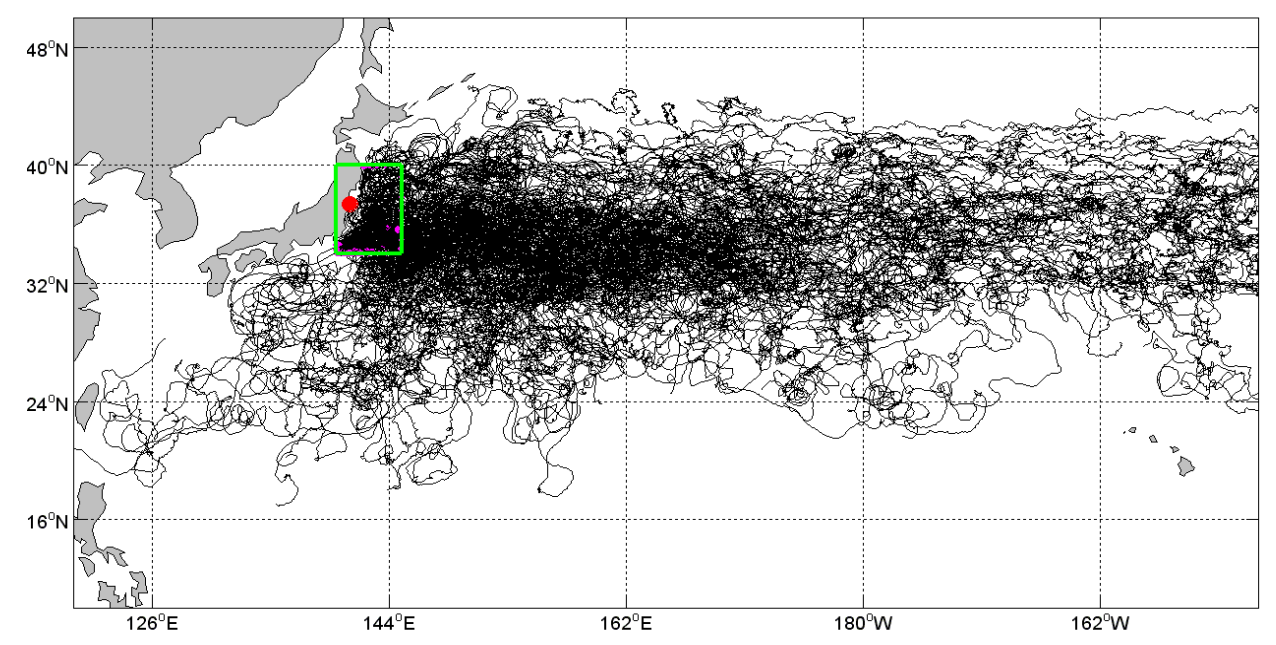

Figure. 3 SVP surface drifting buoys around Fukushima offshore

From Fig. 3 we can see that most of the buoys float out of the rectangular area and continue drifting to east, but several buoys drift to the southwest. We select two typical buoys, one of which flows into East China Sea and the other flows into South China Sea. It takes about 1.5 years of the two buoys to flow from Fukushima to China Sea. Initial analysis show that boundary Kelvin waves, mesoscale eddies and the local seasonal weather system may the main factor leading the two buoys into China Sea. We know that a right-hand boundary is necessary for a Kelvin wave[2,6]. The land-sea distribution makes it possible for the waves to transmit from Japan to the China Sea. The buoys can drift into the China Sea through Kelvin wave. During the Kuroshio flowing from southwest to northeast, several mesoscale eddies roll out of it $[9,10]$. The drift may flow into these eddies and be carried into the China Sea. The main wind directions in April and May are westward, but there may have local wind directions are northeast; it can guide the surface flow spread into China Sea.

Although the radioactive material will be diluted and settled during the horizontal and vertical transports, the nuclear pollutions carried by ocean circulations may affect China Sea marine environment in the long-term, on account of some materials' long half-life periods (see Tab. 1).

\section{Summary}

(1) Through above discussion, we can see that the main flows out of the Fukushima coast are the northward Kuroshio and the southward Oyashio. The two currents meet on the east of Japan and then turn to eastward, forming the north Pacific warm current. The strength of Kuroshio and Oyashio vary during the seasonal changes, the meeting point locate in the north of its average position in spring and summer, and in the south of its average position in autumn and winter. Anyhow, they turn to east in the end. The tracers Lagrange simulation show a similar result. And the SVP are also approving this phenomenon. So the nuclear pollutions carried by the ocean circulation are mostly spreading to eastward in seasonal and annual time scale, they can rarely affect China Sea marine environment.

(2) According to the three-dimensional ocean numerical model results, especially the surface drifting buoy trajectory data, the local currents near the Fukushima coast flow south or southwest. Analysis shows this phenomenon is affected by mesoscale vortex, Kelvin waves and seasonal atmosphere system, etc. The drift buoys found in the rectangular area at the Fukushima offshore can be achieved near the sea on the east of Taiwan, East China Sea and even the northern South China Sea after about 1.5 years.

In addition, the North Pacific subtropical circulation needs more than two years to complete one circle. Although the radioactive material will be diluted and settled in the process, some artificial 
radioactive materials have longer half-life periods than the circle period. Therefore, the nuclear pollutions carried by ocean circulation may affect China Sea marine environment in the long-term.

\section{Acknowledgment}

First and foremost, I would like to show my deepest gratitude to my director, Mr. Nie Feng, a respectable, responsible and resourceful Senior, who has provided me with valuable guidance in every stage of the writing of this paper. This work was supported by the 54 batch of National Postdoctoral fund (No. 2013M540565).

\section{References}

[1] B. Xue-zhi, B. Hong and H. Dun-xin et al. "Cyclonic Recirculation on the left side of the kuroshio in the East China Sea. Journal of Hydrodynamics, Ser. B, 13(3): pp. 133-137, 2011.

[2] R. BLECK, G. HALLIWELL, A.WALLCRAFT, et al. "Hybrid Coordinate Ocean Model”, User's Manual, 2012, 199p.

[3] P. ERIC, E. HURLBURT and O. MARTIN The HYCOM (HYbrid Coordinate Ocean Model) data assimilative system. Journal of Marine Systems, 2007, 65: pp. 60-83.

[4] X. GUO, H. Hukuda, Y. Miyazawa, et al. A Triply Nested Ocean Model for Simulating the Kuroshio Roles of Horizontal Resolution on JEBAR. Journal of Physical Oceanography, 2013.33(1): pp.146-169.

[5] P. ZHENG, P. BAI, W. De-xing, et al. "The remote connection of the Taiwan and Tsushima warm currents". Acta Oceanologica Sinica, 2009, 31(1):pp.1-7.

[6] T. AKIKAWA, H. J OON nd K. D. CHO "The Tsushima Warm Current through Tsushima Straits estimated from ferryboat ADCP data" Journal of Physical Oceanography, 2015, 35: pp.1154-1168.

[7] P. ZHENG, W. De-xing and L. Xiao-pei. "Interannual Variability of Kuroshio Current and Its Effect on the Nearshore Branch in Japan/East Sea". Journal of Hydrodynamic, Ser. B, 2014, 22(3): pp.305-311.

[8] A. ISOBE "Two-layer model on the branching of the Kuroshio southwest of Kyushu". Journal of Physical Oceanography, 2014, 30: pp. 2461-2476.

[9] K. KIM, K. I. CHANG, D. J. KANG, et al. Review of Recent Findings on the Water Masses and Circulation in the East Sea (Sea of Japan). Journal of Oceanography, 2012, 64: pp.721- 735.

[10]N. Mimura, Y. Kazuya, K. Seiki, et al. Damage from the Great East Japan Earthquake and Tsunami - A quick report [J]. Earth and Environmental Science, 2014, 16(7): pp. 803-818. 